\title{
Non-Hodgkin lymphomas - T cell type: Histomorphology and immunohistochemistry
}

\author{
Priavadhana Rajan Prasaad ${ }^{1, *}$, Bhawana Ashok Badhe ${ }^{2}$ \\ ${ }^{\mathbf{1}}$ Assistant Professor, Dept. of Pathology, Sree Balaji Medical College and Hospital, (BIHER), Chennai, Tamil Nadu, \\ ${ }^{2}$ Professor, Dept. of Pathology, Jawaharlal Nehru Institute of Post Graduate Medical Education and Research (JIPMER), \\ Pondicherry, India
}

*Corresponding Author: Priavadhana Rajan Prasaad

Email: drpriarajan@gmail.com

Received: $26^{\text {th }}$ December, 2017

Accepted: $25^{\text {th }}$ April, 2018

\begin{abstract}
Introduction: Non-Hodgkin lymphomas (NHL) comprise a wide variety of neoplasms and are subdivided into two main categories of low grade and high grade lymphomas. The major subtypes among $\mathrm{T}$ cell lymphomas are Peripheral $\mathrm{T}$ cell lymphomas - Not otherwise specified, Anaplastic Large cell lymphoma, ALK positive, Angioimmunoblastic T-cell lymphoma, T lymphoblastic leukemia/lymphoma and Extranodal NK/T cell lymphoma, nasal type. In this study, the histomorphological diagnostic features and the adjunct immunohistochemical expression of various markers are studied in a tertiary care centre in South India.

Materials and Methods: Patients with lymphadenopathy over a period of two years were included in the study. Lymph nodes excised from these patients were routinely processed and examined. After initial examination with H\&E stain, appropriate special stains like Reticulin stain, Periodic Acid Schiff stain and Ziehl-Neelsen stains are performed along with the specific Immunohistochemical markers.

Results: A total of 30 cases were classified as Non-Hodgkin Lymphoma, high grade, T cell type. Peripheral T cell lymphomasNOS is found to be the most common tumors accounting for $40 \%$ of all high grade $\mathrm{T}$ cell NHLs. The next common tumor was Anaplastic Large Cell Lymphoma, ALK positive with $36 \%$ of cases. T lymphoblastic leukemia/lymphoma accounted for $10 \%$ of cases and the rest of the cases were reported as Angioimmunoblastic T-cell lymphoma (7\%) and Extranodal NK/T cell lymphoma, nasal type (7\%).

Conclusion: Appropriate use of immunohistochemistry aids in the sub-classification of lymphomas according to the recommendations of WHO 2008. But, further molecular studies should be performed for confirmation of the subtyping done by immunohistochemistry.
\end{abstract}

Keywords: High grade lymphoma, Anaplastic large cell lymphoma, Peripheral T cell lymphoma.

\section{Introduction}

Non-Hodgkin lymphomas (NHL) comprise a wide variety of neoplasms and are subdivided into two main categories of low grade and high grade lymphomas. The high grade lymphomas can arise either from the B cells or from the $\mathrm{T}$ cells. These high grade lymphomas are very aggressive lymphomas with a poor prognosis and survival rate. $\mathrm{T}$ cell high grade NHLs constitute a wide group with various neoplasms under this category. The major subtypes are Peripheral T cell lymphomas Not otherwise specified (PTCL-NOS), Anaplastic Large cell lymphoma (ALCL), Angioimmunoblastic Tcell lymphoma (AITL), $\mathrm{T}$ lymphoblastic leukemia/lymphoma (T-ALL) and Extranodal NK/Tcell lymphoma, nasal type. In this study, the histomorphological diagnostic features and the immunohistochemical expression of various markers were studied in a tertiary care Centre in South India.

\section{Materials and Methods}

Step I: Histomorphological Analysis: Patients with lymphadenopathy attending the general medicine outpatient department and the surgery out-patient department constituted the study population from the period of August 2009 to August 2011 over a period of two years. The lymph nodes were excised and received in the department of Pathology. These were routinely processed (formalin fixed and paraffin embedded) and 3 microns sections were taken, stained with Hematoxylin and Eosin (H\&E) and examined. After the initial examination with $H \& E$ stain, appropriate special stains like Reticulin stain, Periodic Acid Schiff stain and Ziehl-Neelsen stains were performed along with specific immunohistochemical markers.

Step II: Immunohistochemical Analysis: Immunohistochemistry was carried out in the formalin fixed and paraffin embedded sections after treatment with series of xylene and alcohols. Subsequently, these sections were treated with $3 \% \quad \mathrm{H}_{2} \mathrm{O}_{2}$ solution for blocking the endogenous peroxidase activity. After this, the antigens were retrieved by incubating the sections in the citrate buffer at a $\mathrm{pH}$ of 6 using the Heat Epitope Antigen Retrieval method (HEAR) by pressure cooking. Once the antigens were retrieved the sections were incubated for 60 minutes in the primary antibody solutions and the streptavidin - biotin polymer complex was used to label these antibodies. The final colour reaction product was brown with the use of the 
chromogen Diamino benzidine (DAB) and the results were interpreted as either positive or negative and specified as cytoplasmic, membranous or nuclear staining in the tumor cells. After a morphological diagnosis was made, the following panel of markers were used-CD 3, CD4, CD 8, CD 20, CD 10, CD 15, CD 30, TdT, EMA, ALK protein, EBV LMP, BCL 2 and Ki67 (MIB 1) for the differential diagnosis and sub-categorisation of lymphomas.

\section{Results and Observations}

Epidemiology of $\mathbf{T}$ cell Lymphomas: The total number of lymph node biopsies received in the Department of Pathology during the study period was 378. The various diagnoses given in these 378 lymph nodes are as shown in the Chart 1 .

\section{Chart 1: Lymphnode diagnoses}

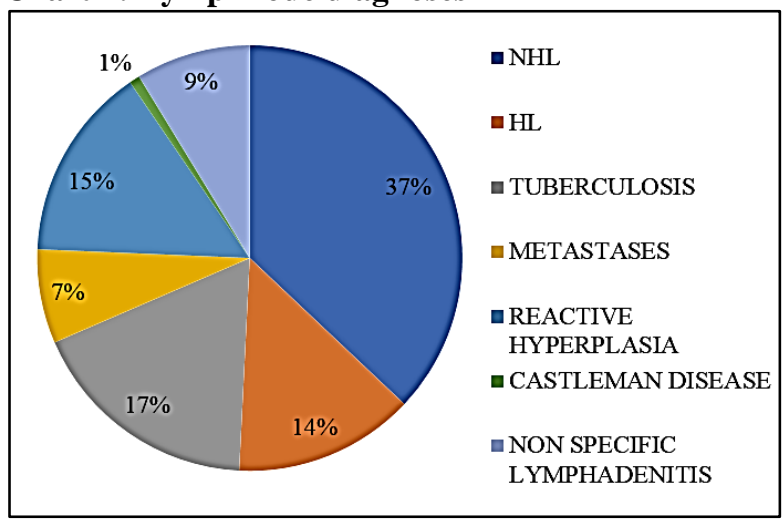

Out of the total 140 cases diagnosed as NonHodgkin Lymphoma, 72 cases were diagnosed as high grade $(51.4 \%)$. This study includes 59 cases from the 72 cases and the 13 cases excluded from the study are due to the unavailability of the tissues for the application of immunohistochemical markers. Among these 59 cases, 30 cases were subclassified as high grade $\mathrm{T}$ cell Non Hodgkin Lymphomas (51\%). The subclassification of $\mathrm{T}$ cell lymphomas is given in Chart 2 .

\section{Chart 2: Distribution of high grade T NHL $\mathbf{N}=30$}

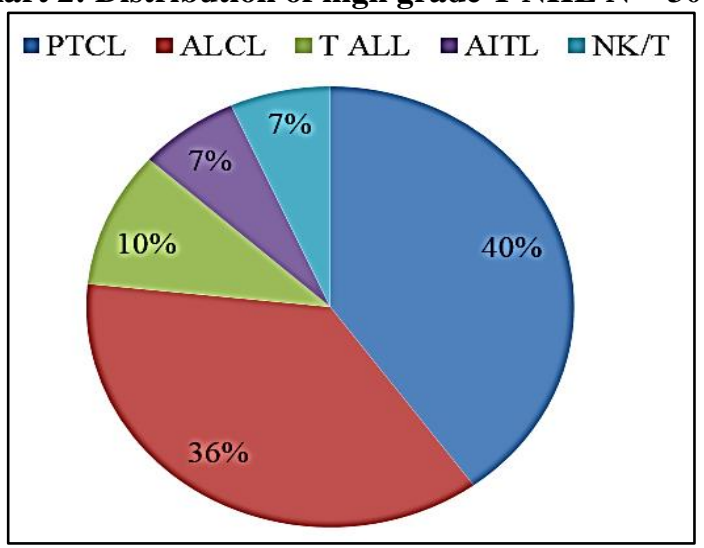

The majority of the $\mathrm{T}$ cell high grade lymphomas were either Peripheral T cell lymphomas-Not otherwise specified (PTCL-NOS) $(40 \%)$ or anaplastic large cell lymphomas (ALCL) (36\%) among all the $\mathrm{T}$ cell lymphomas observed in the study.

Among the PTCLs two common categories were observed namely the PTCL-NOS Fig. 1 accounted for $23 \%$ of the total T cell lymphomas and the PTCL with Lennert's morphology accounting for $17 \%$ of the total T cell lymphomas.

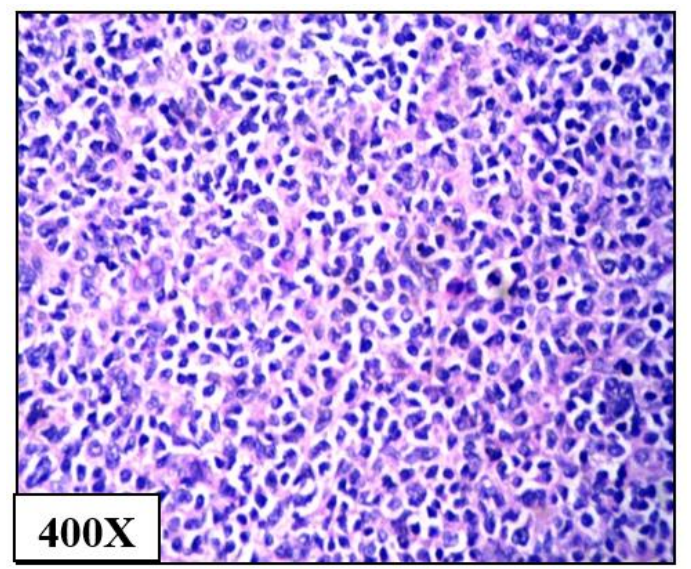

Fig. 1: High power view of peripheral $T$ cell lymphoma

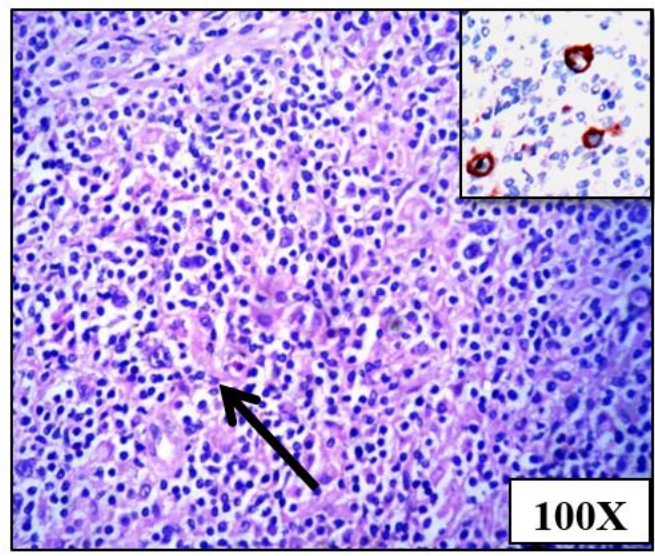

Fig. 2: Low power view of anaplastic large cell lymphoma; Inset - CD 30 positive in large cells; Arrow - large atypical cells

Similarly ALCL's Fig. 2 are subclassified into two distinct entities with clinical and prognostic significance as ALK protein Positive which accounts for $23 \%$ of the total $\mathrm{T}$ lymphomas and Negative accounting for $13 \%$ of the total.

The other types of lymphomas seen in this study are the $\mathrm{T}$ lymphoblastic leukemia/lymphoma accounting for $10 \%$ and the rest by angioimmunoblastic T-cell lymphoma Fig. 3 (7\%) and Extranodal NK/T-cell lymphoma, nasal type. Fig. 4 (7\%). 


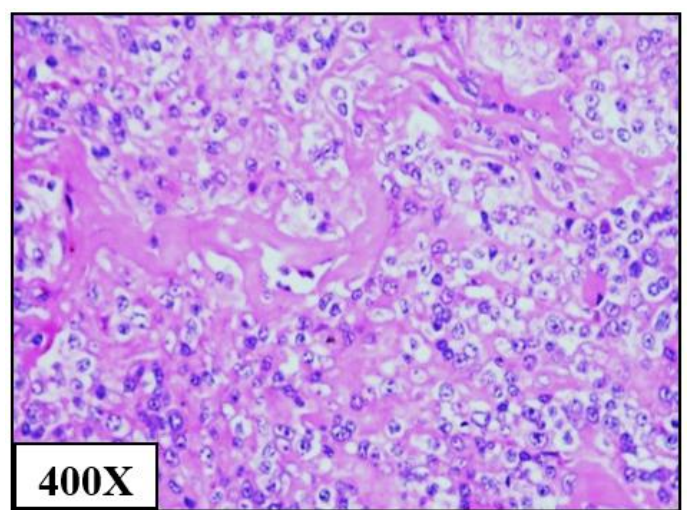

Fig. 3: High power view of Angio-immunoblastic variant of PTCL



Fig. 4: High power view of NK/T cell lymphoma, Nasal Type; Arrow - highlights blood vessel wall infiltrated with atypical lymphoid cells

Age \& Gender Distribution: The male to female ratio in T high grade NHL's is $1.6: 1$ with a slight male preponderance. The age range was from 2-71 with a median age of 45 years. The various age incidences of the lymphomas are shown in the Chart 3.

Chart 3: The median age of the various subsets of high Grade T NHL

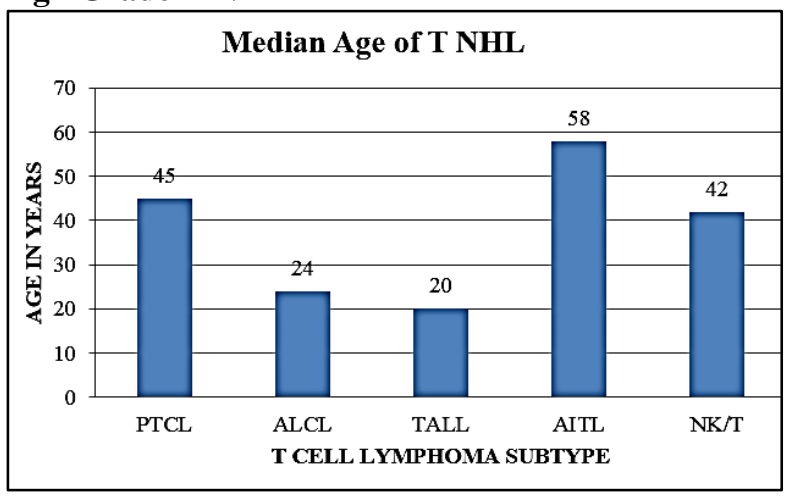

Duration of Symptoms and Tumor Bulk: The high grade $\mathrm{T}$ cell lymphomas presented with a mean duration of symptoms for 3.8 months, with a range of the shortest of 10days to two years. The average size of the mass/ lymphnodes at presentation was $2.6 \times 2 \mathrm{~cm}$.

Staging of Lymphomas: Clinical staging is done for these lymphomas according to the Ann-Arbor Staging system. ${ }^{1}$ and it is found that B symptoms are less commonly seen in Non-Hodgkin lymphomas with only few cases presenting with fever and weight loss being the most common symptoms observed. The stages of presentation of the different $\mathrm{T}$ cell high grade NHLs are shown in Charts $4 \& 5$.

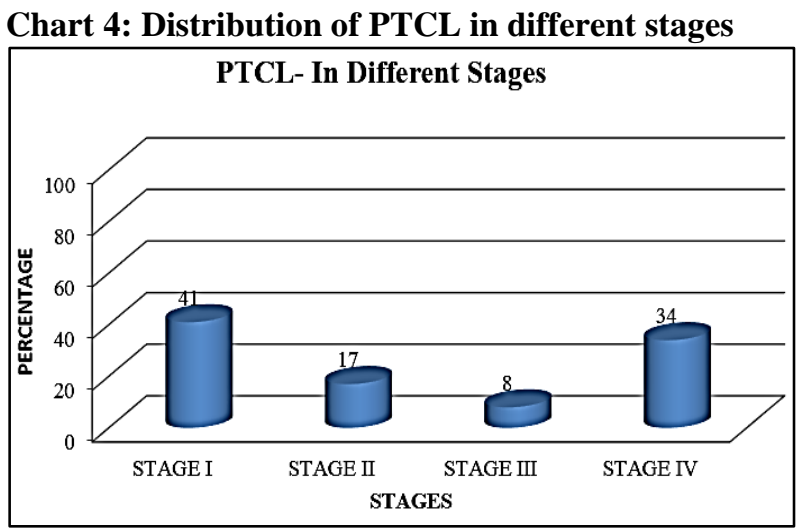

Chart 5: Distribution of ALCL in different stages

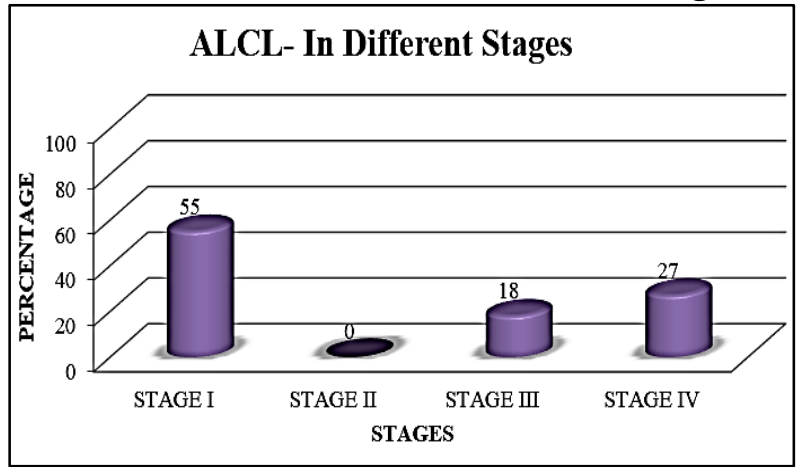

The other subtypes of $\mathrm{T}$ cell lymphomas presented predominantly in the earlier stages. Out of the three $\mathrm{T}$ lymphoblastic leukemia/lymphomas, two of them presented in Stage I disease and one presented in Stage II.

The two Extranodal NK/T-cell lymphoma, nasal type presented in Stage I disease.

Out of the 2 Angioimmunoblastic T-cell lymphomas, one of them presented with Stage I disease and the other presented with bonemarrow involvement (Stage IV).

Immunohistochemical Profile of T Cell NHL'S: The immunohistochemical panel used for the subcategorisation of the $\mathrm{T}$ cell lymphomas and their percentage expression is as shown in Table 1 
Table 1: Immunohistochemical profile of $t$ cell NHLS

\begin{tabular}{|l|c|c|c|c|c|}
\hline IHC & PTCL (\%) & ALCL (\%) & AITL (\%) & TALL (\%) & Extranodal NK/T (\%) \\
\hline CD 3 & 100 & 45 & 100 & 100 & 100 \\
\hline CD 15 & 0 & 9 & 0 & 0 & 0 \\
\hline CD 30 & 42 & 100 & 0 & 0 & 0 \\
\hline EMA & 0 & 100 & 0 & 0 & 0 \\
\hline ALK & 0 & 64 & 0 & 0 & 0 \\
\hline CD 10 & 0 & 0 & 100 & 0 & 100 \\
\hline EBV-LMP & 0 & 0 & 50 & 0 & 30 \\
\hline KI-67 & 30 & 25 & 40 & 85 & \\
\hline
\end{tabular}

CD 3, the lineage marker of the T cells is expressed in all $(100 \%)$ of the PTCLs, AITLs, T-ALLs and Extranodal NK/T cell lymphoma, nasal types. In ALCLs this marker expression is seen only in five out of the 11 cases $(45 \%)$ thus categorising them as ALCLs- $\mathrm{T}$ cell phenotype. The rest six cases did not express this marker in the tumor population and only background reactive population showed positivity, thus subcategorising them as ALCL- Null cell phenotype.

CD 30 expression is seen in all the 11 cases of ALCL $(100 \%)$ and also found expressed in five cases of the PTCL $(42 \%)$ thus categorising the PTCLs into a separate subtype based on immunohistochemical expression as the CD 30 positive PTCLs.

EMA is positive in all cases of ALCL (100\%) and not in other subtypes of $\mathrm{T}$ cell lymphomas.

CD 15 is positive in only one of the ALCL cases (9\%) and was confirmed with the ALK positivity and hence ruling out Hodgkin lymphoma.

ALK protein expression is seen in seven cases of the ALCLs thus defining two distinct subcategories among this group into the ALK positive and ALK negative subtypes. ALK expression is not seen in the other subtypes of the high grade NHLs.

CD 10 is found to be positive in all cases of AITLs $(100 \%)$ but not in other subtypes. EBV-LMP is expressed in one of the two angioimmunoblastic cases $(50 \%)$. This marker is expressed in both cases of Extranodal NK/T-cell lymphoma, nasal type (100\%).

The other markers used are natural killer cell markers, CD 16 and CD 56 which were negative in both the cases of the Extranodal NK/T-cell lymphoma, nasal type.

\section{Discussion}

NHLs arising from the mature $\mathrm{T}$ cells constitute the most heterogeneous group of neoplasms namely the Peripheral T cell lymphomas. The recent WHO 2008 classification has three distinct entities in this category namely the Peripheral T-Cell Lymphoma-Not Otherwise Specified (PTCL-NOS), Angioimmunoblastic T cell lymphoma (AITL) and Anaplastic large cell lymphoma (ALCL). ${ }^{1-10}$ This group represents $10-15 \%$ of the NHLs worldwide with slightly higher incidence in Asian countries. ${ }^{11-14}$ These lymphomas generally pursue an aggressive clinical course with both nodal and extra-nodal manifestations and adverse outcome. ${ }^{15}$

PTCL-NOS is a diagnosis of exclusion. The median age of presentation of this group is in the sixth or seventh decade with $65 \%$ of the patients having stage IV disease and a poor five year survival rate of 20-30. ${ }^{16-}$ 19

Several morphological variants have been described in PTCL-NOS. Variants described in the recent WHO 2008 classification are PTCLlymphoepithelioid variant (Lennert's lymphoma), the interfollicular variant and the $\mathrm{T}$ zone variants..$^{20-23}$

ALCL is a rare lymphoma worldwide with an incidence of $<5 \%$ but represents the most common large cell lymphoma occurring in the pediatric population accounting for $10 \%-15 \%$ of the pediatric/adolescent NHL's compared to the $2 \%$ incidence in adults. ${ }^{24-25}$ Usually, ALCL presents with advanced stage of III/IV with frequent bone marrow and extra-nodal involvement, skin and bone being the most common sites of involvement of systemic ALCL. ${ }^{26}$

Jeffrey et al in their study on ALCL have found several morphological variants in ALCL, with the classical entity $(70 \%)$ characterized by the neoplastic "hallmark" cells and a background polymorphous infiltrate of lymphocytes, plasma cells and eosinophils. $^{25}$ The other variants described are the lympho-histiocytic (5-10\%), small cell (5-10\%) and sarcomatoid variant $(<1 \%)$. Another morphological variant included in the WHO 2008 is the Hodgkin like ALCL.

In their study, they have also expressed the significance of IHC in ALK positive ALCL (50-70\% of ALCL), in that it correlates with the molecular signature of the $t(2,5)$ by having a specific Golgi region of distribution of ALK protein in these cases.

Summers et al in their study of the specific variant small cell ALCL have found that, this variant has a distinctly aggressive course compared to the other variants with prominent lymphadenopathy, leukemic presentation and disseminated disease with frequent skin involvement. ${ }^{27}$

Jhala et al in their study have reported a rare morphologic entity of neutrophil rich ALCL in two HIV positive patients, concluded that this entity should 
be considered in neutrophil rich specimens with otherwise the typical morphology of ALCL and absence of ALK expression. ${ }^{29}$

Jhala et al in their study on 66 patients of ALK positive and ALK negative ALCLs, have found that BCL2 expression has a significant difference and hence prognosis in these two groups with BCL2 being more frequently expressed in the ALK negative group (57\%) compared to the complete absence of its expression in ALK positive ALCL $(0 \%){ }^{31}$

Several prognostic parameters characterize this lymphoma with the expression or the non-expression of the ALK protein uniquely characterized by the $t(2,5)$ with fusion of NPM -ALK genes, playing the most important role. Five year failure free survival studies have shown a better prognosis for those with expression of ALK protein (ALK + ALCL), younger age and a worse prognosis for the ALK - ALCL and those expressing the epithelial membrane Antigen (EMA). ${ }^{30-}$ 36

Berge et al evaluated the role of Epithelial Membrane Antigen (EMA) in 79 cases of ALCLs, HLs, DLBCLs and PTCLs and have concluded that there is no statistical significant difference in the expression of EMA in these tumors. But this marker is seen consistently to be expressed in ALK positive ALCLs compared to the low or nil expression in ALK negative ALCLs and HLs. ${ }^{35}$

The distinction of ALK positive and negative lymphomas with that of the Peripheral T cell lymphoma (PTCL) has been made possible with the appropriate use of IHC markers. Savage et al in their study on 181 patients collected from 22 institutions across the world have emphasized the need for the distinction of ALK negative ALCL from the PTCL - NOS type, since they have found that immunohistochemically as well as in survival studies, the ALK negative group have significant differences. ${ }^{37}$ These express the CD30 antigen strongly and have better five year survival rates (36\%) which were superior compared to the PTCL group (20\%).

In more recent studies, it has been proposed that the diagnosis of ALCL should be revised when EBV markers are positive. But in recent case reports, they have opined that EBV could be expressed in ALCLs and that FISH detection of other EBV markers than EBER could be helpful in actual detection of the viral status in these groups of lymphomas.

Yu zeng and Andrew L Feldman in their study on the genetics of ALCL have studied the role of ALK protein mutations. ${ }^{38}$ They have observed that recurrent chromosomal rearrangements with the ALK protein and its partners namely NPM1 as in $t(2,5)$ contribute to the pathogenesis of ALCL. The other partner genes identified are TPM3, TPM4 and many others. This NPM-ALK fusion protein activates many signal transduction pathways like the JAK/STAT, mTOR,
PI3K/AKT pathway which are important signaling pathways in cell proliferation and survival.

They have also observed that ALK negative tumors consistently express two recurrent genetic abnormalities, one involving the DUSP22 (an inhibitor of $\mathrm{T}$ cell receptor signaling) and the other being TP53 mutations.

\section{Conclusion}

Appropriate use of immunohistochemistry aids in the sub-classification of lymphomas according to the recommendations of WHO 2008. But, further molecular studies should be performed for confirmation of the subtyping done by immunohistochemistry.

\section{References}

1. Swerdlow S.H, Campo E, Harris N.L, Jaffe E.S, Pileri S.A and Stein H., Thiele J, Vardiman J.W. Editors. WHO Classification of Tumors of Hematopoietic and Lymphoid Tissues. IARC: Lyon 2008.

2. Ioachim HL, Ratech H. Nomenclature and classification of lymphomas. In: Ioachim's Lymphnode Pathology. 3rd ed. Philadelphia. Lippincott Williams and Wilkins; 2002. p. 315-24.

3. Gocke CD, Non-Hodgkin's lymphoma. In: Dabbs DJ. Diagnostic Immunohistochemistry. Philadelphia: Churchill Livingstone; 2002. p. 113-134.

4. Armitage JO. A Clinical Evaluation of the International Lymphoma Study Group Classification of NonHodgkin's Lymphoma - By the Non Hodgkin Lymphoma's lymphoma Classification Project. Blood. 1997;89:3909-18.

5. Jaffe ES, Harris NL, Stein H and Isaacson PG. Classification of lymphoid neoplasms: the microscope as a tool for disease discovery. Blood. 2008;112:4384-99.

6. Melnyk A, Rodriguez A, Pugh WC and Cabannillas F. Evaluation of the Revised European - American Lymhoma Classification confirms the clinical relevance of immunophenotype in 560 cases of aggressive NonHodgkin's Lymphoma. Blood. 1997;89:4514-20.

7. Campo E, Swerdlow SH, Harris NL, Pilera S, Stein H and Jaffe ES. The 2008 WHO classification of lymphoid neoplasms and beyond: evolving concepts and practical applications. Blood. 2011;117:5019-32.

8. Jaffe ES. The WHO 2008 classification of lymphomas: implications for clinical practice and translational research. Hematology. 2009.

9. Recommendations for the Reporting of Lymphoid Neoplasms. Association of Directors of Anatomic and Surgical Pathology. Am J Clin Pathol. 2003;119:185-189.

10. Clarke CA and Glaser SL. Changing Incidence of Non Hodgkin Lymphomas in the United States. Cancer 2002. 94: 2015-23

11. Muller AM, Ihorst G, Mertlsmann R and Engelhardt M. Epidemiology of Non Hodgkin Lymphoma (NHL): trends, geographic distribution and etiology. Ann Hematol. 2005;84:1-12.

12. Alexander DD, Mink PJ, Adami HO, Chang ET, Cole P, Mandel JS. The Non Hodgkin Lymphomas: A review of the epidemiologic literature. Int J Cancer. 2007;120:1-39.

13. Naresh KN, Srinivas V and Soman CS. Distribution of various subtypes of Non-Hodgkin's lymphoma in India: a Study of 2773 lymphomas using R.E.A.L and WHO classifications. Ann Oncol. 2000;11:63-6. 
14. Naresh KN, Agarwal B, Sangal BC, Basu DD, Kothari AS and Soman CS. Regional variation in the distribution of subtypes of lymphoid neoplasms in India. Leuk lymphoma. 2002;43:1939-43.

15. Ko OB, Lee DH, Kim SW, Lee JS, Kim S and Huh J, et al. Clinicopathologic characteristics of T cell NonHodgkin's Lymphoma: A single institution experience. Korean J Intern Med. 2009;24:128-134

16. Arrowsmith ER, Macon WR, Kinney MC, Stein RS, Goodman SA and Morgan DS, et al. Peripheral T cell lymphomas: clinical features and prognostic factors of 92 cases defined by the Revised European American Lymphoma classification. Leuk Lymphoma. 2003;44:2419.

17. de Leval L and Gaulard P. Pathobiology and molecular profiling of Peripheral T cell lymphomas. Hematology Am Soc Hematol Educ Program. 2008;272-9.

18. Went P, Agostinelli C, Gallamini A, Piccaluga PP, Ascani S and Sabattini E, et al. Marker expression in Peripheral T cell lymphoma:A proposed clinic-pathologic prognostic score. J Clin Oncol. 2006;24:2472-79.

19. Warnke RA, Jones D and Hsi ED. Morphologic and immunophenotypic variants of Nodal T cell lymphomas and T cell lymphoma mimics. Am J Clin Pathol. 2007;127:511-27.

20. Piccaluga PP, Agostinelli C, Gazolla A, Manny C, Bacci $\mathrm{F}$ and Sabattini E. Prognostic markers in Peripheral T cell lymphoma. Curr Hematol Malig Rep. 2010;5:222-28

21. Kalyan K, Basu DD and Jayanthi S. Immunohistochemical typing of Non-Hodgkin's lymphoma- comparing Working Formulation and WHO classification. Indian J Pathol Microbiol. 2006;49:203-7.

22. Iqbal J, Weisenburger DD, Greiner TC, Vose JM, McKeithan T, Kucuk C. Molecular signatures to improve diagnosis in Peripheral T cell lymphoma and prognostication in angioimmunoblastic T cell lymphoma. Blood. 2010;115:1026-36.

23. de Leval L, Gisselbrecht $\mathrm{C}$ and Gaulard P. Advances in the understanding and management of angioimmunoblastic T cell lymphoma. Br J Hematol. 2010;148:673-89

24. Kinney MC, Higgins RA and Medina EA. Anaplastic Large Cell Lymphoma: Twenty five years of Discovery. Arch Pathol Lab Med. 2011;135:19-43.

25. Jeffrey LM and Kojo SJ. Anaplastic Large Cell lymphoma. Am J Clin Pathol. 2007;127:707-22.

26. Jacobsen E. Anaplastic large cell lymphoma, T/Null Cell type. Oncologist. 2006;11:831-40.

27. Summers TA and Moncur JT. The Small cell variant of Anaplastic Large cell lymphoma. Arch Pathol Lab Med. 2010;134:1706-10.

28. Falini B and Martelli MP. Anaplastic large cell lymphoma: changes in the World Health Organisation classification and perspectives for targeted therapy. Hematologica. 2009;94:897-900.
29. Jhala DN, Medeiros LJ, Lopez-Terrada D, Jhala NC, Krishnan B, Shahab I. Neutrophil Rich Anaplastic Large Cell lymphoma of $\mathrm{T}$ cell lineage- A report of two cases arising in HIV positive patients. Am J Clin Pathol. 2000;114:478-482.

30. Delsol G, Brugieres L, Gaulard P, Espinos E and Lamant L. Anaplastic large cell lymphoma, ALK positive and anaplastic large cell lymphoma ALK negative. Hematology Meeting Reports. 2009;3:51-57.

31. Jhala DN, Medeiros LJ, Lopez-Terrada D, Jhala NC, Krishnan B, Shahab I. Differential expression of BCL 2 family proteins in ALK positive and ALK negative Anaplastic Large Cell lymphoma of T/ Null Cell lineage. American J Pathol. 2001;159:527-35.

32. Attarbaschi A, Mann G, Rosolen A, Williams D, Uyttebroeck A, Marky I. Prognosis in childhood anaplastic large cell lymphoma- limited stage I disease is not necessarily indicative of an exvellent prognosis. Blood. 2011;117:5616-19.

33. ten Berge RL, Meijer CJ, Dukers DF, Kummer JA, Bladergroen BA, Vos W, et al. Expression levels of apoptosis-related proteins predict clinical outcome in anaplastic large cell lymphoma. Blood. 2002;4540-6

34. Rassidakis GZ, Goy A, Medeiros LJ, Jiang Y, Thomaides A, Remache Y. Prognostic significance of MUC-1 expression in systemic anaplastic large cell lymphoma. Clin Cancer Res. 2003;9:2213-20.

35. ten Berge RL, Snijdewint FG, von Mensdorff-Pouilly S, Poort-Keesom RJ, Oudejans JJ and Meijer JW. MUC-1 (EMA) is preferentially expressed by ALK positive anaplastic large cell lymphoma in the normally glycosyalated or only partly hypoglycosylated form. $J$ Clin Pathol. 2001;54:933-39.

36. Stein H, Foss HD, Durkop H, Marafioti T, Delsol G and Pulford K, et al. CD $30+$ Anaplastic large cell lymphoma: a review of its histopathologic, genetic and clinical features. Blood. 2000;96:3681-95.

37. Savage KJ. Prognosis and primary therapy in Peripheral T cell lymphomas. Hematology. 2008;280-8.

38. Yu Zeng and Andrew L Feldman. Genetics of Anaplastic Large cell Lymphoma. Leuk Lymphoma. 2016;57(1):2127.

How to cite this article: Prasaad P. R, Badhe B. A. Non-Hodgkin lymphomas - $\mathrm{T}$ cell type: Histomorphology and immunohistochemistry. Indian J Pathol Oncol. 2018;5(4):636-641. 